\title{
Histological outcomes after focal high-intensity focused ultrasound and cryotherapy
}

\author{
Taimur T. Shah ${ }^{1,4} \oplus$ - Veeru Kasivisvanathan ${ }^{1,3,4} \cdot$ Charles Jameson $^{5} \cdot$ Alex Freeman $^{5}$ • \\ Mark Emberton ${ }^{1,2,3} \cdot$ Hashim U. Ahmed ${ }^{1,3}$
}

Received: 4 February 2015 / Accepted: 13 April 2015 / Published online: 6 May 2015

(C) The Author(s) 2015. This article is published with open access at Springerlink.com

\begin{abstract}
Introduction Focal therapy has increasingly become an accepted treatment option for patients with localised prostate cancer. Most follow-up protocols use a mixture of protocol biopsies or "for cause" biopsies triggered by a rising PSA. In this paper, we discuss the histological outcomes from these biopsies and their use in guiding subsequent management and trial development.

Methods We conducted a literature search and reviewed the post-treatment biopsy results from studies on focal HIFU and focal cryotherapy. We subsequently reviewed the results of three recently published consensus statements released discussing many of the issues concerning focal therapy.

Results Research suggests that 1 in 5 of all post-treatment biopsies after focal therapy are positive. However, the majority of these seemed to be from the untreated portion of the gland or met criteria for clinically insignificant disease. The histological outcomes from focal therapy are promising and confirm its effectiveness in the short to
\end{abstract}

Taimur T. Shah

taimur.shah@doctors.org.uk; taimur.shah@ucl.ac.uk

1 Division of Surgery and Interventional Science, Urology

Research Group, UCL, Room 4.23, 4th Floor, 132

Hampstead Road, London NW1 2PS, UK

2 NIHR UCLH/UCL Comprehensive Biomedical Research Centre, London, UK

3 Department of Urology, UCLH NHS Foundation Trust, London, UK

4 Department of Urology, Whittington Hospital NHS Trust, London, UK

5 Department of Histopathology, UCLH NHS Foundation Trust, London, UK medium term. Furthermore re-treatment is possible whilst maintaining a low-side-effect profile.

Conclusion Debate is ongoing about the clinical significance of various levels of residual disease after focal therapy and the exact threshold at which to call failure within a patient who has had focal therapy.

Keywords Focal therapy $\cdot$ Prostate cancer $\cdot$ Minimally invasive therapy $\cdot$ High-intensity focussed ultrasound · HIFU · Cryotherapy · Histology · Pathology · Trial design

\section{Introduction}

The emergence of focal therapy as a potential and major shift in the way we manage localised prostate cancer is a highly debated area of research in uro-oncology. Whilst the concept of the index lesion being the primary driver for progressive prostate cancer seems attractive and its ablation seems logical, the wider acceptance of focal therapy, even using such ablative modalities as cryotherapy and high-intensity focused ultrasound (HIFU) seems limited and questioned. This is especially an issue when regulatory approvals are being considered for device approvals or an approval for an additional indication including the focal ablation of a lesion [1, 2]. However, focal therapy has been increasing in popularity, which is highlighted by the increasing number of focal cryotherapy cases registered in the Cryo Online Database (COLD). The number of focal procedures performed per year has increased from 1 in 1999 to 293 in 2007 [3-5].

Currently, there is an array of treatment modalities being evaluated for the focal treatment of localised prostate cancer. Whilst the two in pole position are HIFU and cryotherapy, having been used for focal therapy for a longer period 

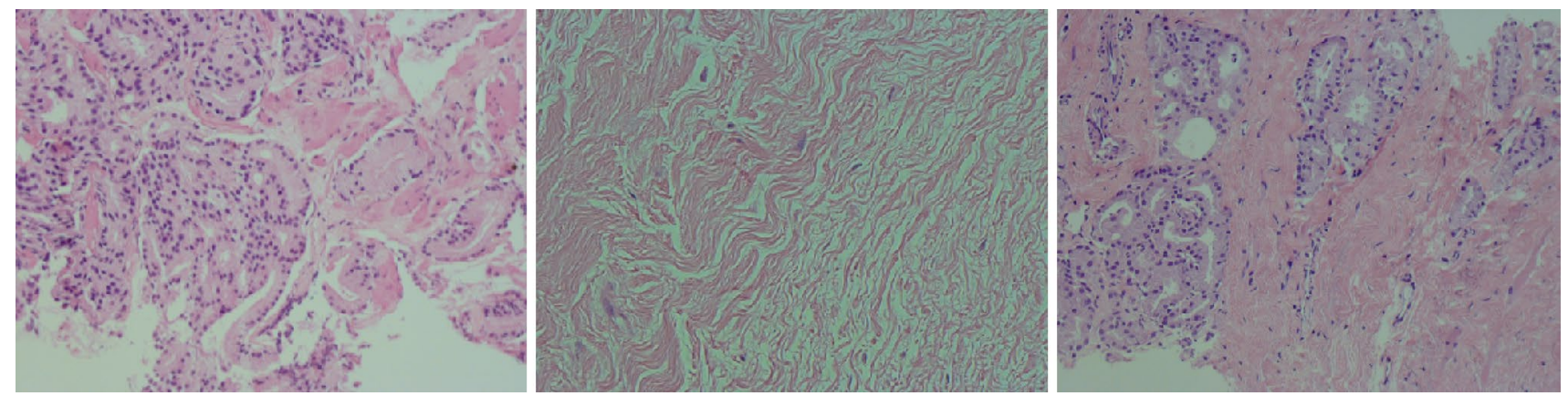

Fig. 1 Left image shows a Gleason $3+4$ adenocarcinoma, middle image shows typical changes after HIFU with no discernible glands, viable cells or tumour with presence of fibrosis and elastotic collagen, right image shows recurrent adenocarcinoma after HIFU

of time, others under investigation include photodynamic and vascular-targeted photodynamic therapy (PDT/VTP) [6, 7], irreversible electroporation [8], radiofrequency ablation [9], magnetic thermotherapy [10], convective thermal water vapour [11], injectable toxins [12] and focal brachytherapy [13]. The aim of all these technologies is to treat the index lesion within the prostate whilst leaving behind healthy non-cancerous tissue or non-significant disease.

One of the significant areas of debate relates to how success or failure of focal therapy is assessed, regardless of which ablative modality is being delivered. The arguments for and against a number of outcome measures follow a common theme in all studies and considerations given for acceptance and approval for focal therapy. Established prostate-specific antigen (PSA) follow-up criteria used for whole-gland treatments such as ASTRO, Phoenix and the Stuttgart criteria, are difficult to apply to a patient after focal therapy as the untreated normal prostatic tissue will continue to secrete PSA. PSA kinetics and PSA nadir may play a role as PSA secretion from the cancerous lesion is larger than healthy prostate tissue, and Ahmed et al. [14] noted an $80 \%$ decrease in PSA at 3 months after focal HIFU. This decrease persisted at 12 months. The more solid endpoints of metastases and death would require over a decade of follow-up due to the long natural history of even clinically significant prostate cancer. Insisting on such outcomes for changes in clinical practice or regulatory approvals would inevitably stifle innovation. Not only would these findings have limited external validity, reported after 10-15 years but would be prohibitively expensive and resource heavy. Other outcome measures, such as histological outcomes, are clearly needed.

In this paper, we aim to first discuss the existing stateof-the-art with respect to histological outcomes after focal therapy and how best to interpret these in clinical practice. The literature is mainly based on the two most widely used modalities of HIFU and cryotherapy. Secondly, we will discuss histological endpoints from a trial design point of view that might be met with wider acceptance or at the very least, form the premise for further debate.

\section{Histological changes on radical prostatectomy specimens}

\section{HIFU}

In one of the very first proof of concept studies, Van Leenders et al. assessed the histological changes in unilateral HIFU in men who subsequently underwent a radical prostatectomy (RP) 7-12 days later. This study was set up in the early evaluation of HIFU using the ablate-excise study model to demonstrate that ablation can be achieved, whilst the investigators did not set out to fully ablate all tissue. Macroscopically, well-demarcated circular and ellipsoid lesions were seen. Microscopically, cell necrosis was seen within the core of the lesion, however, and not surprisingly, this was incomplete in six out of nine lesions. Haemorrhage with hyperplastic epithelium and reparative changes was also seen at the borders of the lesion [15].

Napoli et al. [16] performed a similar study in five patients with RP performed 7-14 days after HIFU treatment. They also found extensive coagulative necrosis but with no viable tumour within or at the boundaries of the treated lesion. Subsequently, over time, there is development of fibrosis and elastotic collagen (Fig. 1).

Both these studies observed the presence of multi-focal lesions outside of the treated lesion and thus emphasise the need for accurate pre-operative staging and disease localisation.

\section{Cryotherapy}

In 1991 Onik et al. [17] showed complete coagulative necrosis and accurate visualisation of the ice ball under transrectal ultrasound (TRUS) in six dogs treated with 
cryotherapy. Larson et al. examined exactly the effects of this technique for cryotherapy in five patients who were already scheduled to go undergo salvage prostatectomy 2-3 weeks later. Histologically, squamous metaplasia of glandular epithelium with haemorrhage and a zone of coagulative necrosis and devitalisation was seen spreading from a central core [18]. However, similar to the HIFU studies, incomplete cell death on occasions has also been noted with cryotherapy [19].

\section{Post-treatment biopsies}

Earlier work with protocol-driven TRUS biopsies in 167 out of 176 patients treated with whole-gland cryoablation showed persistent tumour in $38 \%$. However, in this study, the exact histological data and thus clinical significance of the biopsy results were not mentioned [19]. Similarly, Crouzet et al. [20] performed post-treatment biopsies in 774 patients from cohort of 1002 patients treated with whole-gland HIFU procedures. Overall, $37 \%$ of these biopsies were positive.

Donnelly et al. also performed a randomized control trial (RCT) where 244 men with $\mathrm{T} 2 / 3$ prostate cancer were randomised to either whole-gland cryotherapy or radiotherapy. At a median follow-up of 100 months, they found no difference in disease progression between the two groups. They also found a higher number of positive biopsies at 36 months in the radiotherapy arm (28.9 compared $-7.7 \%$ with cryotherapy) [21].

Reviewing the results of eight studies assessing focal cryotherapy, 98/391 (25\%) of all post-treatment biopsies were positive [3,22-28] (Table 1). No information on grade or location for the 42 positive biopsies from the COLD registry paper by Ward et al. was available. Thus, reviewing the results from the remaining papers shows that $86 \%$ (48/56) of positive biopsies were either from an untreated portion of the prostate or met with criteria of insignificant lesions (Gleason 3, $\leq 2$ cores positive).

Six of these studies mentioned the initial method of diagnosis and only two used transperineal mapping biopsies whilst all the others relied on TRUS biopsies for preoperative planning. The two studies that did use mapping biopsies had a 13-19\% post-procedure positive biopsy rate $[22,27]$.

Similarly, from the six studies assessing biopsies after focal HIFU, $22 \%$ (39/175) were positive [14, 29-33] (Table 2). Excluding the four positive biopsies from the paper by Muto et al., as no data on location or histological grade were available, $63 \%(22 / 35)$ of the positive biopsies were either insignificant or from the untreated part of the gland (Table 2).

However, results such as these have to be interpreted with caution as they are from a heterogeneous group of for-cause, protocol-driven, bilateral or targeted biopsies. It would be expected that for-cause biopsies would be more likely to be positive as they are performed for a clinical suspicion of recurrence. However, studies that only conduct for-cause biopsies, means that those with stable PSAs are selectively not subjected to verification biopsies after treatment. If protocol biopsies are performed then these should be aimed at determining initial treatment success and thus should be targeted at the treated lesion. Additionally, performing biopsies of untreated areas is not assessing treatment success but rather the accuracy of pre-operative assessment.

Eleven studies mentioned the subsequent management of 62 patients in total who had a positive post-treatment biopsy. $61 \%$ (38/62) elected for redo focal therapy and $31 \%(19 / 62)$ chose active surveillance (AS), whilst $8 \%$ $(5 / 62)$ had either external beam radiotherapy (EBRT), RP or androgen deprivation therapy (ADT). The ability to retreat with curative intent without significant additional morbidity is a major advantage of focal therapy over whole-gland treatments such as RP or EBRT.

Berge et al. [34] recently published the results of 130 patients undergoing a second HIFU procedure of which 19 underwent a second redo session and one had a third session. Overall, this group was formed from a cohort of 359 patients and thus represented the $36.2 \%$ who needed repeat treatment based on biochemical, histological or imaging (mpMRI) failure. No cancer-specific deaths were reported in this group. Fifty-six men (43\%) did fail a second time. Forty underwent TRUS biopsy and 22 were positive. Their results also showed that side effects were not greatly increased with an increase in pad usage from 2.7 to $9 \%$ $(p<0.001)$ and no effect on potency $(p=0.9)$. Blana et al. [35] had also previously shown a minimal impact on quality of life with a second session of HIFU.

A significant portion chose AS, and there is indirect evidence to suggest that this may be an acceptable option for patients with positive post-treatment biopsies. Data from an AS series of 450 patients diagnosed on TRUS biopsy (and therefore approximately $30 \%$ of whom would have been true intermediate risk) shows a 10-year cancer-specific survival of $97.2 \%$ with only five deaths. A recent update which now included 993 men with up to 16-year followup showed a 15-year actuarial cancer-specific survival of $94.3 \%$ [36, 37]. Another indirect source of evidence is studies assessing the outcomes from patients who have post-radiotherapy positive biopsies. 21-32 \% of patients may have positive biopsies. The results show that although patients with a positive biopsy have a poorer outcome their 5-year biochemical disease-free survival (bDFS) was still high at $83.3-93.8 \%$ compared to $97.5 \%$ for those with a negative biopsy [38]. In contrast though, Zelefsky assessed 10 -year oncological outcomes and found that the group 


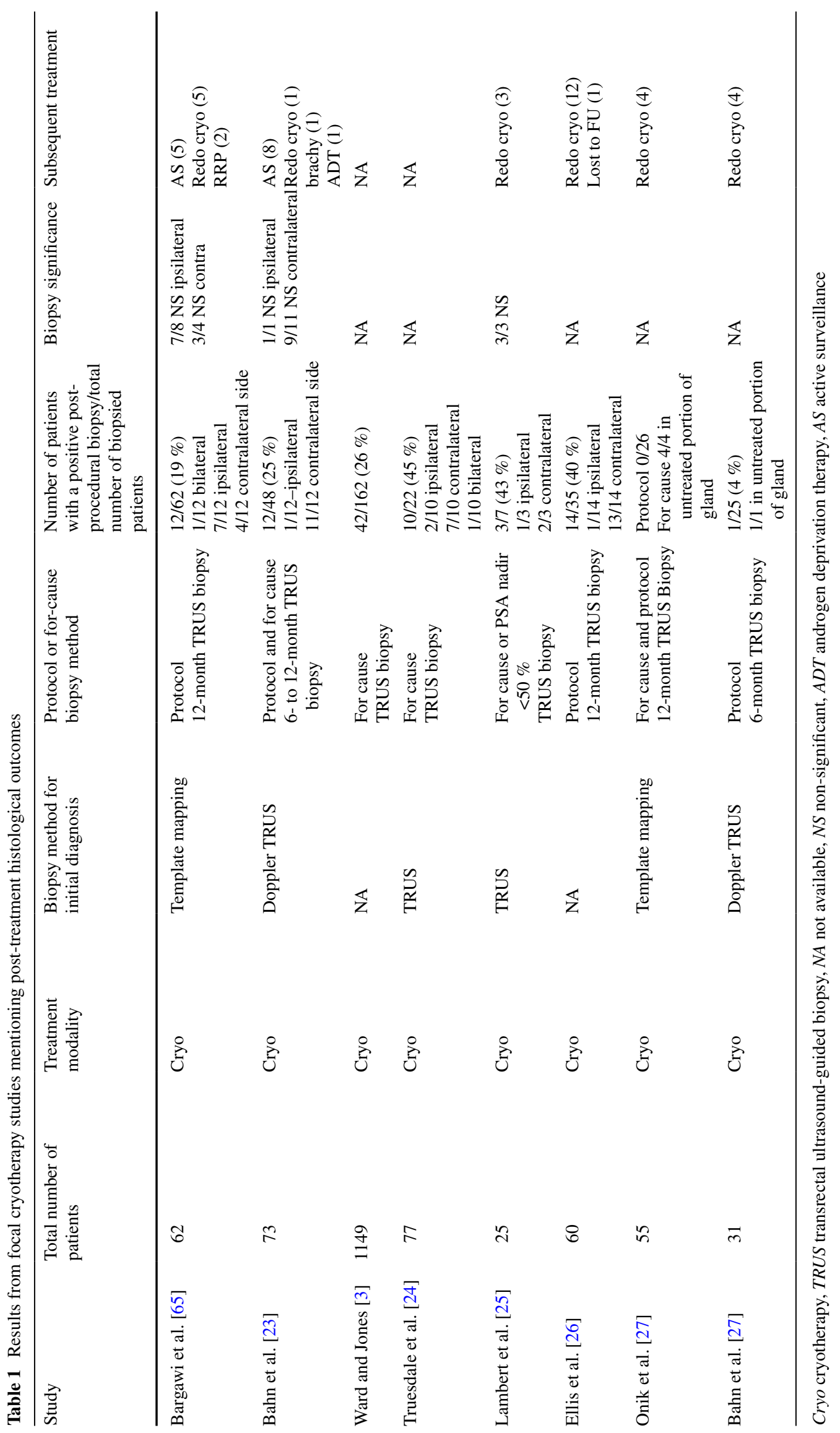




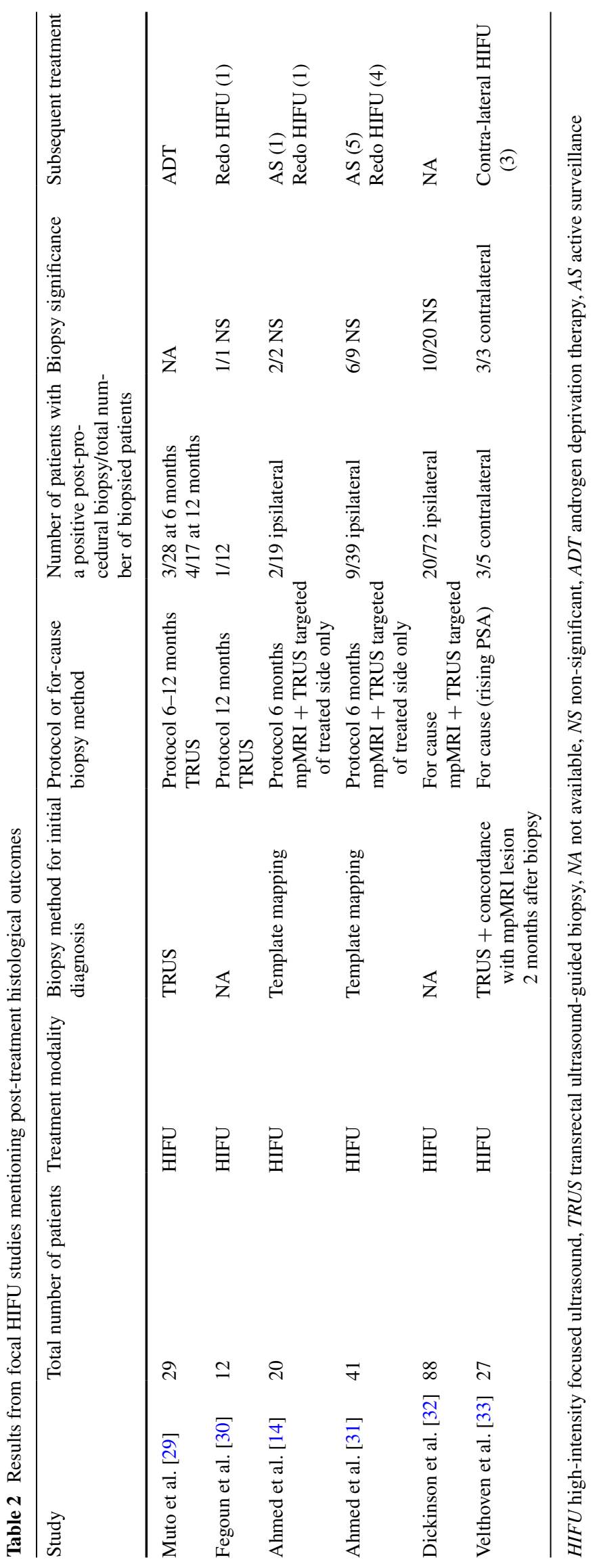


with positive biopsies had only a $3 \%$ PSA relapse-free survival and a $69 \%$ metastases-free survival compared to 59 and $90 \%$, respectively, for patients with negative biopsies. Zelefsky did have a third group of patients who had positive biopsies showing severe treatment effect. This group did not have a significantly different outcome when compared to those with negative biopsies [39]. Thus, a positive biopsy has to be reviewed carefully before a clinical significance can be implied. Similarly, a positive surgical margin after RP does not always convey a poorer outcome [40].

\section{Discussion and focal therapy trial design}

Knowing this data, the questions that need be asked before designing an appropriate trial are "when to biopsy?", "How to biopsy?" and "How to interpret the results?".

It is accepted that a well-designed RCT is high-level evidence upon which treatment decisions can be based. However, designing an appropriate prospective trial to assess the follow-up of patients after focal therapy carries its own challenges. Successfully randomizing men to different surgical treatments in prostate cancer can be challenging.

The initial entry point would be from diagnosis of disease. The methodology for diagnosis is important. Preoperative TRUS biopsy has been shown to be inaccurate particularly when assessing patients for focal therapy. For instance, $23 \%$ of patients having a template biopsy after a previous TRUS biopsy had upgrading, whilst $60 \%$ were found to have bilateral disease [41]. Systematic transperineal and MRI-guided biopsies have been shown to be equally accurate in detecting cancer $[42,43]$. Thus, a similar principle should be applied to post-treatment biopsies. Most still perform systematic TRUS biopsies after treatment that may under-sample the treated area and lead to detection of insignificant disease from untreated tissue.

To improve upon, these ultrasound-MRI fusion techniques have been developed. There seems to be potential benefit of this technique over systematic TRUS biopsies with $67 \%$ more significant disease detected; however, the benefit is less clear when comparing cognitive MRI targeting versus a fusion technique [44-46]. Wysock et al. [45] did not show a significantly increased cancer detection rate between the two techniques, but did find improved accuracy for smaller lesions with fusion biopsies. Whilst Cool et al. [46] showed a $100 \%$ increased accuracy for sampling a clinically significant tumour with fusion targeting versus cognitive. Five millimetres of transperineal mapping biopsies is arguably still the gold standard though, with up to $95 \%$ accuracy when compared to RP specimens $[47,48]$. Nonetheless, these require a general anaesthetic and due to the higher number of cores taken have a potentially higher side effect profile.
Subsequently, the clinical significance of disease needs to be reviewed before any treatment is offered. Gleason 6 disease has been shown to rarely if ever lead to metastases and thus death [49-51]. Similarly, metastases are very rare with tumour volume $<0.5 \mathrm{cc}$ [52]. Combining this data from results from AS series where few deaths occur, it can be reasoned that low-volume Gleason 6 disease may not benefit from interventional treatment [36]. Thus, the ideal focal therapy candidate is likely to be men with Gleason 7 and above disease or high-volume Gleason 6 cancer.

These same factors play a role following focal therapy. If a patient's disease has been accurately classified preoperatively, then follow-up can consist of imaging and biopsies only targeted to the treated area. As previously mentioned, although various criteria exist for follow-up, all effectively conclude that a rising PSA can be used to guide further investigation for recurrence and timing of the posttreatment biopsy. An additional factor to consider is the use of mpMRI to follow up treated lesions and thus triggers a biopsy if residual or recurrent disease is detected.

After focal ablation, there is a residual inflammation, necrosis and eventual fibrosis. Along with some of the data in our own series, Biermann et al. [53] found that detection of grading of cancer in biopsies taken 6 months post-HIFU was possible (unlike with radiotherapy); thus, it seems reasonable that biopsies can be performed at least as early as 6 months.

With respect to mpMRI a consensus meeting found that $77 \%$ of panellists felt that this is a reliable tool for followup [54]. From the series of 42 men by Ahmed et al., nine patients were found to have a positive mpMRI, seven of whom had subsequent positive biopsies. Overall, there is some evidence to support the use of mpMRI as a method for follow-up, with it appearing to form an important part of multi-modal follow-up [55-58].

Subsequently, the clinical significance of post-procedural biopsies needs to be considered. There is limited evidence to suggest that low-grade low-volume residual intreatment field cancer behaves in a similar way to patients with primary disease of the same pathology. Similarly, there is no data on the natural history of secondary lowrisk lesions. As mentioned previously, the safest option would be to apply the same principles as for AS and follow up these patients with repeated imaging with biopsies as deemed necessary. From the reviewed cryotherapy and HIFU papers, redo focal treatment and AS appear to be the most common management options selected for patients with positive biopsies.

We subsequently reviewed the results of three recently published consensus statements released discussing many of these issues concerning focal therapy (Table 3) [59-61]. All consensus meetings and expert opinions are considered as level five evidence, and their findings should be 


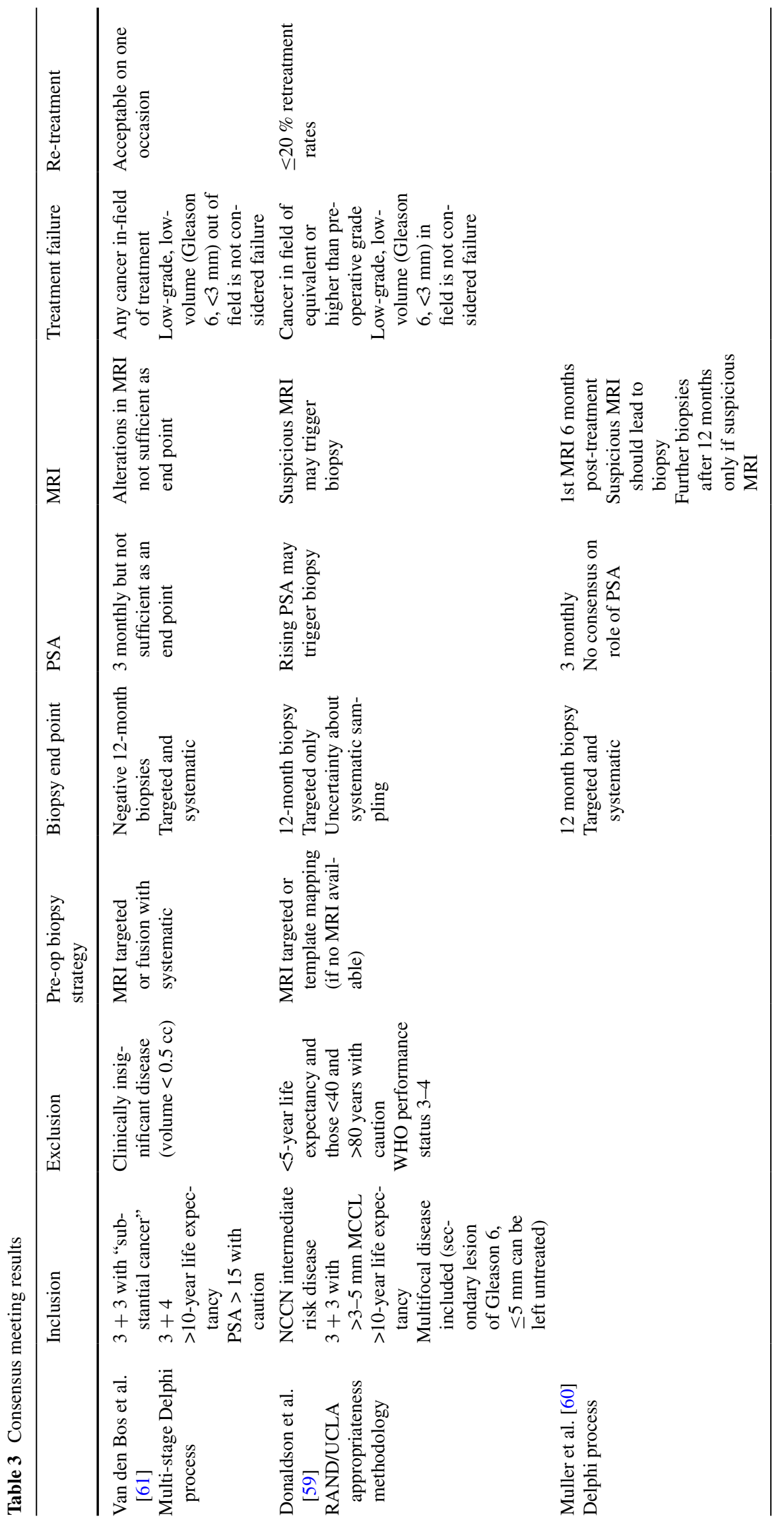


considered with this in mind. However, in the absence of high-level evidence, they present important opinions from experts in the field and highlight areas of uncertainty.

Donaldson et al. and Van den Bos et al. separately discussed inclusion and exclusion criteria and both consensus groups agreed that high-volume Gleason $6(>5 \mathrm{~mm}$ MCCL) and Gleason 7 disease are the optimal candidates. They discussed the importance of accurate pre-operative assessment using MRI-targeted or fusion biopsy methods prior to offering a patient treatment. They recommend protocol biopsies at 12 months post-treatment and that a rising PSA or suspicious MRI should also trigger a biopsy. The ultimate end point was a negative 12-month biopsy. However, there was differing opinion on whether these should only be targeted or also systematic or whether residual low-grade and low-volume cancer is considered treatment failure. Donaldson et al. mentioned that biopsies should be targeted rather than systematic in order to reduce sampling of untreated tissue, whilst Muller et al. (another consensus group) commented that systematic biopsies are useful for surveillance of the untreated gland. Re-treatment was considered acceptable and Donaldson et al. commented that overall re-treatment rate should be below $20 \%$ since it was argued that retreatment was a positive attribute of the strategy.

The final point to consider when designing such a trial is the comparator arm. The two most common radical wholegland treatments are radiotherapy or RP. Neither has been assessed head-to-head in a RCT. Retrospective reviews have shown surgery to have better oncological outcomes; however, even with the best matching, bias can never be fully excluded $[62,63]$.

The role of focal therapy will ultimately help decide the most appropriate comparator arm. We feel that the current role of focal therapy is similar to that of tissue preservation in almost all other solid organ cancers (bar ovarian), in that, it leads to a minimal decrease in quality of life for the patient whilst providing acceptable cancer control. At least in the short to medium term, the data suggest that focal therapy may be meeting these aims. However, few studies have long-term follow-up and a systematic review showed a varying bDFS of between $86.2 \%$ at 8 years and $60 \%$ at 5 years [64].

Thus, in the first instance, patients with localised intermediate- to high-risk disease should be compared to those undergoing radical treatment with either EBRT or RRP. The aim of this study would be to show non-inferiority for short- to medium-term oncological outcomes with a superior side effects profile. Repeat treatments are possible with focal therapy, and a second treatment would not be considered a treatment failure unless there was a significant reduction in the two outcome measures mentioned.

\section{Conclusion}

Research suggests that one in five of all post-treatment biopsies after focal therapy is positive. However, the majority of these seemed to be from the untreated portion of the gland or met criteria for clinically insignificant disease. The histological outcomes from focal therapy are promising and confirm its effectiveness in the short to medium term. Furthermore, re-treatment is possible whilst maintaining a low side effect profile. Debate is ongoing about the clinical significance of various levels of residual disease after focal therapy and the exact threshold at which to call failure within a patient who has had focal therapy.

Acknowledgments M. Emberton and H. U. Ahmed would like to acknowledge funding from the Medical Research Council (UK), the Pelican Cancer Foundation Charity, Prostate Cancer UK, St Peters Trust Charity, Prostate Cancer Research Centre the Welcome Trust, National Institute of Health Research-Health Technology Assessment Programme and the US National Institute of Health-National Cancer Institute. M. Emberton receives funding in part from the UK National Institute of Health Research UCLH/UCL Comprehensive Biomedical Research Centre. M. Emberton and H. U. Ahmed receive funding from USHIFU, GSK and Advanced Medical Diagnostics for clinical trials. M. Emberton is a paid consultant to Steba Biotech and USHIFU. Both have previously received consultancy payments from Oncura/GE Healthcare and Steba Biotech.

Conflict of interest V. Kasivisvanathan is funded by a Doctoral Research Fellowship from the National Institute for Health Research. The views expressed in this publication are those of the authors and not necessarily those of the NHS, the National Institute for Health Research or the department of health.

Ethical standard This paper does not contain any direct clinical or patient data. All the studies reviewed had appropriate ethics committee approval and have therefore been performed in accordance with the ethical standards laid down in the 1964 Declaration of Helsinki and its later amendments.

Open Access This article is distributed under the terms of the Creative Commons Attribution 4.0 International License (http://creativecommons.org/licenses/by/4.0/), which permits unrestricted use, distribution, and reproduction in any medium, provided you give appropriate credit to the original author(s) and the source, provide a link to the Creative Commons license, and indicate if changes were made.

\section{References}

1. FDA (2014) Executive summary: Ablatherm ${ }^{\circledR}$ integrated imaging high intensity focused ultrasound (HIFU). Meeting of the Gastroenterology and Urology Devices Panel. http://www.fda. gov/downloads/AdvisoryCommittees/CommitteesMeetingMaterials/MedicalDevices/MedicalDevicesAdvisoryCommittee/ Gastroenterology-UrologyDevicesPanel/UCM406819.pdf

2. Valerio M, Emberton M, Ahmed HU (2014) Re: Jarow et al: Drug and device development for localized prostate cancer: report of a Food and Drug Administration/American Urological 
Association public workshop (Urology 2014;83:975-979). Urology 84(3):732-733

3. Ward JF, Jones JS (2012) Focal cryotherapy for localized prostate cancer: a report from the national Cryo On-Line Database (COLD) registry. BJU Int 109(11):1648-1654

4. Ahmed HU (2009) The index lesion and the origin of prostate cancer. N Engl J Med 361(17):1704-1706

5. Ahmed HU et al (2012) Do low-grade and low-volume prostate cancers bear the hallmarks of malignancy? Lancet Oncol 13(11):e509-e517

6. Moore CM, Azzouzi AR, Barret E, Villers A, Muir GH, Barber NJ, Bott S, Trachtenberg J, Arumainayagam N, Gaillac B, Allen C, Schertz A, Emberton M (2014) Determination of optimal drug dose and light dose index to achieve minimally invasive focal ablation of localised prostate cancer using WST11-vasculartargeted photodynamic (VTP) therapy. BJU Int. doi:10.1111/ bju. 12816

7. Moore CM et al (2011) Light penetration in the human prostate: a whole prostate clinical study at $763 \mathrm{~nm}$. J Biomed Opt 16(1):015003

8. Valerio M et al (2014) A prospective development study investigating focal irreversible electroporation in men with localised prostate cancer: nanoknife electroporation ablation trial (NEAT). Contemp Clin Trials 39(1):57-65

9. Shariat SF et al (2005) Pilot study of radiofrequency interstitial tumor ablation (RITA) for the treatment of radio-recurrent prostate cancer. Prostate 65(3):260-267

10. Johannsen M et al (2007) Thermotherapy of prostate cancer using magnetic nanoparticles: feasibility, imaging, and threedimensional temperature distribution. Eur Urol 52(6):1653-1661

11. Mynderse L, Dixon C, Cabanas C, Rijo Cedano E, Huidobro C, Larson T (2014) Novel convective thermal water vapor therapy for prostate cancer: uniquely suited to zonal anatomy of the prostate-preliminary gross and microscopic findings using ex vivo and acute in vivo, surgical ablative studies. In: 7th international symposium on focal therapy and imaging in prostate and kidney cancer. Pasadena, LA, USA

12. Meschenmoser $\mathrm{K}$ et al (2013) Targeting cancer with a bi-functional peptide: in vitro and in vivo results. In Vivo 27(4):431-442

13. Polders DL, Steggerda M, van Herk M, Nichol K, Witteveen T, Moonen L, Nijkamp J, van der Heide UA (2015) Establishing implantation uncertainties for focal brachytherapy with I-125 seeds for the treatment of localized prostate cancer. Acta Oncol. doi:10.3109/0284186X.2014.995312

14. Ahmed HU et al (2011) Focal therapy for localized prostate cancer: a phase I/II trial. J Urol 185(4):1246-1254

15. Van Leenders GJ et al (2000) Histopathological changes associated with high intensity focused ultrasound (HIFU) treatment for localised adenocarcinoma of the prostate. J Clin Pathol 53(5):391-394

16. Napoli A et al (2013) Real-time magnetic resonance-guided high-intensity focused ultrasound focal therapy for localised prostate cancer: preliminary experience. Eur Urol 63(2):395-398

17. Onik G et al (1991) Percutaneous transperineal prostate cryosurgery using transrectal ultrasound guidance: animal model. Urology $37(3): 277-281$

18. Larson TR et al (2000) In vivo interstitial temperature mapping of the human prostate during cryosurgery with correlation to histopathologic outcomes. Urology 55(4):547-552

19. Koppie TM et al (1999) The efficacy of cryosurgical ablation of prostate cancer: the university of California, San Francisco experience. J Urol 162(2):427-432

20. Crouzet $\mathrm{S}$ et al (2014) Whole-gland ablation of localized prostate cancer with high-intensity focused ultrasound: oncologic outcomes and morbidity in 1002 patients. Eur Urol 65(5):907-914
21. Donnelly BJ et al (2010) A randomized trial of external beam radiotherapy versus cryoablation in patients with localized prostate cancer. Cancer 116(2):323-330

22. Barqawi $\mathrm{AB}$ et al (2014) Targeted focal therapy for the management of organ confined prostate cancer. J Urol 192(3):749-753

23. Bahn D et al (2012) Focal cryotherapy for clinically unilateral, low-intermediate risk prostate cancer in 73 men with a median follow-up of 3.7 years. Eur Urol 62(1):55-63

24. Truesdale MD et al (2010) An evaluation of patient selection criteria on predicting progression-free survival after primary focal unilateral nerve-sparing cryoablation for prostate cancer: recommendations for follow up. Cancer J 16(5):544-549

25. Lambert EH et al (2007) Focal cryosurgery: encouraging health outcomes for unifocal prostate cancer. Urology 69(6):1117-1120

26. Ellis DS, Manny TB Jr, Rewcastle JC (2007) Focal cryosurgery followed by penile rehabilitation as primary treatment for localized prostate cancer: initial results. Urology 70(6 Suppl):9-15

27. Onik G et al (2007) "Male lumpectomy": focal therapy for prostate cancer using cryoablation. Urology 70(6 Suppl):16-21

28. Bahn DK et al (2006) Focal prostate cryoablation: initial results show cancer control and potency preservation. J Endourol 20(9):688-692

29. Muto S et al (2008) Focal therapy with high-intensity-focused ultrasound in the treatment of localized prostate cancer. Jpn J Clin Oncol 38(3):192-199

30. Fegoun ABE et al (2011) Focal therapy with high-intensity focused ultrasound for prostate cancer in the elderly. A feasibility study with 10 years follow-up. Int Braz J Urol 37(2):213-219 discussion 220-2

31. Ahmed HU et al (2012) Focal therapy for localised unifocal and multifocal prostate cancer: a prospective development study. Lancet Oncol 13(6):622-632

32. Dickinson L, Ahmed HA, McCartan N, Freeman A, Kirkham A, Allen C, Hindley R, Emberton M (2012) Medium term outcomes following primary focal therapy using HIFU for localised prostate cancer. BJU Int 109(Suppl S7):6

33. Van Velthoven $\mathrm{R}$ et al (2014) Primary zonal high intensity focused ultrasound for prostate cancer: results of a prospective phase IIa feasibility study. Prostate Cancer 2014:756189

34. Berge V et al (2014) Morbidity associated with primary high intensity focused ultrasound and redo high intensity focused ultrasound for localized prostate cancer. J Urol 191(6): 1764-1769

35. Blana A et al (2006) Morbidity associated with repeated transrectal high-intensity focused ultrasound treatment of localized prostate cancer. World J Urol 24(5):585-590

36. Klotz L et al (2010) Clinical results of long-term follow-up of a large, active surveillance cohort with localized prostate cancer. J Clin Oncol 28(1):126-131

37. Klotz L et al (2015) Long-term follow-up of a large active surveillance cohort of patients with prostate cancer. J Clin Oncol 33(3):272-277

38. D'Alimonte L, Helou J, Sherman C, Loblaw A, Chung HT, Ravi A, Deabreu A, Zhang 1, Morton G (2014) The clinical significance of persistent cancer cells on prostate biopsy after high dose-rate brachytherapy boost for intermediate-risk prostate cancer. Brachytherapy. doi:10.1016/j.brachy.2014.10.003

39. Zelefsky MJ et al (2008) Influence of local tumor control on distant metastases and cancer related mortality after external beam radiotherapy for prostate cancer. J Urol 179(4):1368-1373 discussion 1373

40. Yossepowitch O et al (2014) Positive surgical margins after radical prostatectomy: a systematic review and contemporary update. Eur Urol 65(2):303-313 
41. Onik G, Miessau M, Bostwick DG (2009) Three-dimensional prostate mapping biopsy has a potentially significant impact on prostate cancer management. J Clin Oncol 27(26):4321-4326

42. Radtke JP, Kuru TH, Boxler S, Alt CD, Popeneciu IV, Huettenbrink C, Klein T, Steinemann S, Bergstraesser C, Roethke M, Roth W, Schlemmer HP, Hohenfellner M, Hadaschik BA (2015) Comparative analysis of transperineal template saturation prostate biopsy versus magnetic resonance imaging targeted biopsy with magnetic resonance imaging-ultrasound fusion guidance. $\mathrm{J}$ Urol 193(1):87-94. doi:10.1016/j.juro.2014.07.098

43. Kasivisvanathan $\mathrm{V}$ et al (2013) Transperineal magnetic resonance image targeted prostate biopsy versus transperineal template prostate biopsy in the detection of clinically significant prostate cancer. J Urol 189(3):860-866

44. Siddiqui MM et al (2013) Magnetic resonance imaging/ultrasound-fusion biopsy significantly upgrades prostate cancer versus systematic 12-core transrectal ultrasound biopsy. Eur Urol 64(5):713-719

45. Wysock JS et al (2014) A prospective, blinded comparison of magnetic resonance (MR) imaging-ultrasound fusion and visual estimation in the performance of MR-targeted prostate biopsy: the PROFUS trial. Eur Urol 66(2):343-351

46. Cool DW et al (2015) Evaluation of MRI-TRUS fusion versus cognitive registration accuracy for MRI-targeted, TRUS-guided prostate biopsy. AJR Am J Roentgenol 204(1):83-91

47. Crawford ED et al (2013) Clinical-pathologic correlation between transperineal mapping biopsies of the prostate and three-dimensional reconstruction of prostatectomy specimens. Prostate 73(7):778-787

48. Hu Y et al (2012) A biopsy simulation study to assess the accuracy of several transrectal ultrasonography (TRUS)-biopsy strategies compared with template prostate mapping biopsies in patients who have undergone radical prostatectomy. BJU Int 110(6):812-820

49. Ross HM et al (2012) Do adenocarcinomas of the prostate with Gleason score (GS) $</=6$ have the potential to metastasize to lymph nodes? Am J Surg Pathol 36(9):1346-1352

50. Eggener SE et al (2011) Predicting 15-year prostate cancer specific mortality after radical prostatectomy. J Urol 185(3):869-875

51. Haffner MC et al (2013) Tracking the clonal origin of lethal prostate cancer. J Clin Investig 123(11):4918-4922

52. Bostwick DG et al (1993) Staging of early prostate cancer: a proposed tumor volume-based prognostic index. Urology 41(5):403-411

53. Biermann $\mathrm{K}$ et al (2010) Histopathological findings after treatment of prostate cancer using high-intensity focused ultrasound (HIFU). Prostate 70(11):1196-1200
54. Muller BG et al (2014) Role of multiparametric magnetic resonance imaging (MRI) in focal therapy for prostate cancer: a Delphi consensus project. BJU Int 114(5):698-707

55. Punwani S et al (2014) Prostatic cancer surveillance following whole-gland high-intensity focused ultrasound: comparison of MRI and prostate-specific antigen for detection of residual or recurrent disease. Br J Radiol 2012(85):720-728

56. Kim CK et al (2008) MRI techniques for prediction of local tumor progression after high-intensity focused ultrasonic ablation of prostate cancer. AJR Am J Roentgenol 190(5):1180-1186

57. Del Vescovo R et al (2013) Dynamic contrast-enhanced MR evaluation of prostate cancer before and after endorectal highintensity focused ultrasound. Radiol Med 118(5):851-862

58. Rouviere O et al (2010) Prostate cancer transrectal HIFU ablation: detection of local recurrences using $\mathrm{T} 2$-weighted and dynamic contrast-enhanced MRI. Eur Radiol 20(1):48-55

59. Donaldson IA, Alonzi R, Barratt D, Barret E, Berge V, Bott S, Bottomley D, Eggener S, Ehdaie B, Emberton M, Hindley R, Leslie T, Miners A, McCartan N, Moore CM, Pinto P, Polascik TJ, Simmons L, van der Meulen J, Villers A, Willis S, Ahmed HU (2015) Focal therapy: patients, interventions, and outcomes-a report from a consensus meeting. Eur Urol 67(4):771777. doi:10.1016/j.eururo.2014.09.018

60. Muller BG, van den Bos W, Brausi M, Fütterer JJ, Ghai S, Pinto PA, Popeneciu IV, de Reijke TM, Robertson C, de la Rosette JJ, Scionti S, Turkbey B, Wijkstra H, Ukimura O, Polascik TJ (2015) Follow-up modalities in focal therapy for prostate cancer: results from a Delphi consensus project. World J Urol 14(2):e829-e829b. doi:10.1016/s1569-9056(15)60818-1

61. van den Bos W et al (2014) Focal therapy in prostate cancer: international multidisciplinary consensus on trial design. Eur Urol 65(6):1078-1083

62. Cooperberg MR et al (2010) Comparative risk-adjusted mortality outcomes after primary surgery, radiotherapy, or androgen-deprivation therapy for localized prostate cancer. Cancer 116(22):5226-5234

63. Zelefsky MJ et al (2010) Metastasis after radical prostatectomy or external beam radiotherapy for patients with clinically localized prostate cancer: a comparison of clinical cohorts adjusted for case mix. J Clin Oncol 28(9):1508-1513

64. Valerio $M$ et al (2014) The role of focal therapy in the management of localised prostate cancer: a systematic review. Eur Urol 66(4):732-751

65. Barqawi $\mathrm{AB}$ et al (2014) Targeted focal therapy in the management of organ confined prostate cancer. J Urol 192:749-753 